\title{
La cohésion lexicale en phrase nominale avec un nom sans déterminant
}

\author{
Yumi TAKAGAKI* \\ Université préfectorale d'Osaka, Japon
}

\begin{abstract}
Résumé. On considérera la façon dont la cohésion textuelle est réalisée lexicalement à partir du cas des phrases nominales dont le nom en position frontale n'a pas de déterminant ( nom nu»). Nous montrerons que si les noms nus dans la phrase nominale sont en règle générale abstraits, ils peuvent être concrets et que dans tous les cas ils conservent pleinement leur nature substantive. Au nombre des relations qu'entretient ce nom avec le cotexte antérieur, deux cas sont prépondérants : la répétition et la reprise par un hyperonyme. Nous nous proposons de rendre compte des autres cas en distinguant trois types d'enchaînement: rétrospectif, présentatif et prospectif. L'enchaînement présentatif se distingue en ce que (i) le nom nu actualise un nouvel élément, (ii) la phrase est le plus souvent courte et (iii) l'insertion d'un élément devant le nom nu est possible.
\end{abstract}

\begin{abstract}
Lexical cohesion in nominal sentences that include nouns without determiners. We will consider the way in which textual cohesion is achieved lexically in the case of nominal sentences with a noun in the frontal position that has no determiner ("bare noun"). We will show that, while the bare noun in the nominal sentence is generally abstract, it can be concrete, and that in any case, it retains its full substantive nature. Among the relationships that this noun has with the previous context, two cases are predominant: repetition and replacement by a hypernym. We propose to report on the other cases by distinguishing three types of sequencing: retrospective, presentative, and prospective. The presentative sequence differs in that (i) the bare noun updates a new element, (ii) the sentence is usually short and (iii) it is possible to insert an element in front of the bare noun.
\end{abstract}

\section{Introduction}

L'objectif de cet article est d'examiner de quelle façon la cohésion textuelle peut être réalisée, sans recours à des procédés syntaxiques, par des moyens lexicaux qui assurent la connectivité entre les séquences. La cohésion grammaticale se réalise à partir de la connectivité inter-, intra- ou trans-phrastique, temporelle et référentielle (exophorique ou endophorique). La cohésion lexicale s'établit à partir de la continuité sémantique, fondée sur l'isotopie et sur la relation entre les items lexicaux. Soit les mêmes unités lexicales sont

\footnotetext{
*takagaki@1c.osakafu-u.ac.jp
} 
reprises, soit on procède par substitution en exploitant des relations telles que la synonymie, l'antonymie, l'hyponymie, l'hyperonymie ou la métonymie.

Notre étude se concentrera sur les noms. En français, le nom est presque toujours accompagné d'un déterminant qui assigne au vocable une caractérisation définie ou indéfinie. Le plus souvent, cet agencement syntaxique crée automatiquement une cohésion. Sauf en cas d'expression générique, le groupe nominal avec article défini se trouve lié au contexte, quel que soit le contenu sémantique du nom. Autrement dit, une unité lexicale sans déterminant ne suffit pas pour établir un lien de cohésion. On voudrait en montrer cependant la possibilité, toute marginale qu'elle soit.

L'observation portera sur des cas où l'absence d'article alterne avec l'emploi de l'article défini et de l'article indéfini. Certaines occurrences sont liées à des positions syntaxiques particulières inscrites en position argumentale, dont entre autres, le verbe être suivi d'un nom de métier, avoir pour $\mathrm{N}$, changer de $\mathrm{N}$ et il y a $\mathrm{N}$. Une autre construction sans déterminant se distingue par sa productivité : la phrase nominale.

Examinons la construction averbale incluant une assertion. Dans l'exemple (1), la phrase qui commence par Situation comique est une phrase nominale, dont le nom frontal est nu. Dépourvu de déterminant, le groupe nominal est connecté au cotexte précédent d'une manière exclusivement lexicale. (Dans tous les exemples, c'est nous qui mettons en gras ou soulignons.)

(1) - Ne vous excusez pas, madame, je vous ai avertie que mon histoire est de celles qui font rire, et que j'en connaissais mieux que personne la niaiserie et l'absurdité. Oui, tout cela est burlesque. Mais, croyez-moi si je vous dis que, dans la réalité, ce ne fut pas drôle. Situation comique en apparence, et qui, par la force des choses, demeure comique, mais situation affreuse.

(Maurice Leblanc, Les huit coups de l'horloge)

En (2), on relève deux phrases nominales. La première qui commence par Couvercle a, tout comme en (1), une partie qualificative longue. La seconde est composée d'un seul mot: Résultat.

(2) La terre absorbe environ $50 \%$ de l'énergie que lui envoie le soleil. Cette énergie permet à notre planète de se réchauffer. Mais pour qu'elle remplisse effectivement son rôle, il faut l'empêcher de s'échapper. C'est là le rôle dévolu à un "couvercle" constitué de vapeur d'eau et de gaz. Couvercle indispensable qui agit un peu comme une serre pour des plantes: sans cet "effet de serre", la température moyenne de notre planète descendrait à $-18^{\circ}$ ! Mais point trop n'en faut. Car les gaz issus des activités de l'homme viennent renforcer et faire grossir cette couverture naturelle. Résultat : sous la serre, la chaleur augmente. De plus en plus... A retenir : c'est donc l'homo sapiens et non la nature qui est à l'origine du réchauffement de la terre.

(France 2, Dossier, « La conférence de Kyoto », 12-19 décembre 1997)

Si le lien entre deux phrases sans cohésion syntaxique n'est pas propre aux phrases nominales, cette diversité d'emplois, sans rapport avec l'absence d'article dans les locutions figées, nous a incitée à choisir cette structure pour examiner les conditions dans lesquelles se produit une cohésion exclusivement lexicale.

Dans la section 2, nous définirons notre objet d'étude et dans la section 3 nous présenterons quelques travaux en rapport avec ce sujet. Dans la $4^{\mathrm{e}}$ section, nous montrerons en partant d'un exemple les possibilités de commutation dans le groupe nominal, dans la $5^{\mathrm{e}}$ nous exposerons quelques caractéristiques de la phrase nominale à nom nu pour envisager sa structure et dans la $6^{\mathrm{e}}$ nous examinerons la relation au cotexte antérieur de cette 
construction afin de mettre en évidence comment la cohérence s'établit avec le nom sans déterminant.

\section{La définition du nom nu}

Selon Riegel, Pellat et Rioul (1994 : 151), le déterminant se définit comme « le mot qui doit nécessairement précéder un nom commun pour constituer un groupe nominal bien formé. » De ce fait, ils distinguent un groupe nominal minimal constitué de [déterminant + nom] et un groupe nominal étendu qui est une expansion du groupe nominal minimal par l'addition d'éléments facultatifs tels que (i) l'adjectif ou le groupe adjectival épithète, (ii) le syntagme prépositionnel complément du nom, (iii) la subordonnée relative complétant un antécédent nominal et (iv) la subordonnée complétive et sa réduction infinitive. Or, nous avons montré en (1) et en (2) qu'un nom dépourvu de déterminant peut être syntaxiquement bien formé. Si Riegel, Pellat et Rioul (1994) prévoient une série de cas pour expliquer l'absence de déterminant, ils ne précisent pas véritablement ce qui constitue le caractère bien formé d'un groupe nominal lorsque le déterminant est absent.

Dans cette contribution, nous désignerons comme nom $n u$ un nom dépourvu de déterminant lexicalement réalisé, qu'il s'agisse d'articles (le, un, des, $d u \ldots$ ), de déterminants possessifs (mon, ton, son...), de démonstratifs (ce, cet, cette, ces), d'interroexclamatifs (quel), et de quelques déterminants dits indéfinis comme certain, chaque, quelques, plusieurs... Ces déterminants ne sont pas cumulables. Ce caractère exclut les expressions comme autre, divers, tout qui peuvent être employées avec un article défini ou indéfini (e.g. un autre fait, les diverses questions, toutes les choses).

Si un nom nu est accru par un ou plusieurs qualificatifs, par exemple, la désignation $n u$ sera conservée pour autant qu'il est sans déterminant.

\section{Le cadre de référence}

De nombreuses études ont paru qui se proposent de rendre compte de l'absence de déterminant. Sans prétention à l'exhaustivité, nous citerons Guillaume (1919), Pottier (1962), Bally (1965), Furukawa (1978, 1986), Moignet (1981), Picabia (1983, 1986, 1991), Anscombre (1986a, 1986b, 1991a, 1991b), Noailly (1990), Giry-Schneider (1991), Kupferman (1991), Riegel, Pellet et Rioul (1994), Naganuma (2004), Leeman (2004), Benetti (2008), Buvet (2009), Grevisse et Goosse (2016). Certains postulent l'existence d'un article zéro (e.g. Guillaume 1919, Anscombre 1986b, Benetti 2008), d'autres parlent seulement d'une absence d'article (e.g. Furukawa 1978 et Naganuma 2004). Nous ne reprendrons pas les termes de ce débat, mais nous exploiterons la distinction proposée par Giry-Schneider (1991). Même si sa classification ne recoupe pas exactement la nôtre, nous en retenons le principe en nous focalisant sur les cas où l'absence du déterminant admet la commutation avec un déterminant.

Concernant la phrase nominale, les études de référence de Lefeuvre (1999a, 1999b, 2000, 2005) examinent les cotextes environnants pour révéler la structure informationnelle de la construction. Nous reprendrons dans notre analyse ses distinctions sur le regard rétrospectif/prospectif ( $c f$. Lefeuvre 2005). Pourtant ces travaux n'ont pas inclus de développement spécifique sur la fonction textuelle du nom nu dans cette construction.

Sur les questions de cohésion, nous prendrons appui sur les travaux d'Adam (1999, 2011, 2017, 2018) pour la conception du liage et de la séquence. Nous considérons avec Adam (2017) que la condition de liage est en grande partie déterminée par l'orientation argumentative de l'énoncé. Cette position nous amène à examiner le cotexte environnant de la phrase nominale ainsi que l'aspect argumentatif de cette construction. 
Quant à l'absence de liage explicite, Charolles (1994) observe plusieurs cas de cohérence sans cohésion : il relève que le lecteur a tendance à établir un lien logique, notamment de cause à conséquence. Mais comme il ne traite pas la cohésion liée au nom nu dans la phrase nominale, il reste à examiner sa relation au cotexte.

Dans cet article, plusieurs exemples sont repris à certains des auteurs cités ci-dessus : la source est mentionnée pour chaque occurrence. Souvent, une seule phrase était citée, abstraction faite du cotexte. Comme l'examen de la cohésion textuelle requiert des séquences plus longues, nous avons restitué le texte original où la phrase était incluse.

\section{La commutabilité}

Dans la phrase nominale, le nom en position frontale peut apparaître avec ou sans déterminant. Un exemple de commutation possible se trouve dans les exemples (3) et (4) cités par Naganuma (2004). D'après lui, devant les noms nus problème en (3) et explication en (4), l'article défini et l'indéfini sont tous deux insérables même si sous leur forme nue, la formulation réfère à un emploi figé.

Willy sauvé... malgré lui

L'orque vedette de la Warner dépérissait dans son bassin.

A grands frais, on l'a emmenée retrouver les siens en Islande.

Pas sûr qu'elle s'adapte

Quatorze millions de dollars pour remettre un cétacé en liberté ! C'est le coût de la grande opération écolo-médiatique menée le 9 septembre par les défenseurs de Keiko, l'orque vedette du film Sauvez Willy. Le cétacé de 4,5 tonnes, qui dépérissait dans un aquarium de Newport (Oregon), sur la côte ouest des EtatsUnis, a donc été transféré dans un container rempli d'eau de mer, à bord d'un avion-cargo de l'US Air Force, jusqu'aux îles Vestmann, au sud de l'Islande, non loin de l'endroit où il avait été capturé voilà vingt ans. Après dix heures de voyage, il a été relâché dans un grand bassin aux parois de plastique transparent, aménagé en pleine mer, où il est censé se réadapter pendant quelques mois à la vie sauvage, avant d'être définitivement libéré.

Problème : l'orque a passé la quasi-totalité de ces vingt ans en captivité, et les spécialistes doutent de sa capacité de réadaptation. Capturé bébé en 1979 au large de l'Islande, le cétacé a grandi dans un parc d'attractions de Mexico, où les producteurs de la Warner l'ont repéré pour en faire la vedette de Sauvez Willy. En 1994, après le succès mondial du film, le magazine Life révèle la triste existence de l'orque, qui s'étiole dans son aquarium étriqué, atteinte de maladies de peau et de problèmes cardiaques.

(L'Express, Par Charles Gilbert, 17 septembre 1998, $<$ https://www.lexpress.fr/informations/willy-sauve-malgre-lui_630237.html>, consulté le 12 décembre 2017, partiellement cité par Naganuma 2004)

(4) Informé le mardi 13 octobre que la prochaine discussion sur le Pacs viendrait à l'Assemblée les 7 et 8 novembre, Robert Hue s'est exclamé, tout joyeux : "Ça tombe bien, ça m'arrange ! » Explication : c'est à ces dates-là que doit se tenir la convention sur l'Europe du PC, qui s'annonce délicate, et le secrétaire national est ravi de pouvoir y échapper.

(Le Nouvel Observateur, 1772, 22 octobre 1998, cité par Naganuma 2004)

Avec l'article défini, la cohésion est assurée par l'emploi du déterminant. Avec l'article indéfini serait soulignée l'introduction d'un nouvel élément. Sans déterminant, en dehors du contenu sémantique, aucun indice grammatical n'indique la relation de ce nom nu au 
cotexte antérieur. La commutabilité semble moins restreinte avec l'indéfini, probablement en raison de sa valeur prédicative. Même si dans de nombreux cas le remplacement par les articles définis/indéfinis n'est pas d'évidence, la possibilité d'une commutation du nom nu dans la position frontale de la phrase nominale demeure.

\section{Quelques caractéristiques sémantico-syntaxiques}

Nous analyserons maintenant quelques aspects sémantiques et syntaxiques de la phrase nominale avec un nom nu.

\subsection{Abstrait et concret}

Voici, en (5), une liste de noms nus attestés en phrase nominale.
accueil, acquiescement, arrivage, banderole, certitude, changement, choses, complication, conclusion, condamnation, condition, confession, conflit, conséquence, consolation, coquetterie, couvercle, craquement, désordre, détail, diversité, dommage, enchevêtrement, enjeu, erreur, exemple, explication, fait, forme, fugue, fureur, gain, gens, glose, innovation, intuition, ironie, lacune, lieu, livre, métier, motif, mystère, odeur, opuscule, paradoxe, peine, perplexité, personnalités, pétards, phénomène, point, précaution, preuve, problème, programme, propos, protestation, question, refus, récit, répit, résultat, résumé, revirement, risque, sanction, sérénité, silence, situation, souveraineté, spectacle, stupeur, terreur, thème, trahison, valeur, vérité

La liste est loin d'être exhaustive ; elle récapitule les occurrences recueillies dans divers textes. En l'absence de prescriptions sémantiques contraignantes, la construction s'avère très productive. Elle diffère sur ce point de la construction similaire Il y $a+$ nom nu où seul le nom abstrait est possible.
a. Livre fermé.
$(c f .(35))$
b. *Il y a livre fermé.

Néanmoins, nous relevons beaucoup de déverbaux (condamnation, consolation, craquement, innovation, protestation...) ainsi que des noms impliquant un contenu (conclusion, explication, fait, enjeu, résultat, preuve...). Les noms attestés sont le plus souvent abstraits, sans que soient exclus les noms concrets comme le montrent couvercle (2), infra, récit (21), gens (22), glose et exemple (26), banderoles et pétards (14) et livre (35).

Ces noms nus en phrases nominales peuvent être au pluriel - infra, conclusions (8), confessions (11), complications (12), peines et investigations (13) et banderoles et pétards (14). Dans la liste citée en (5) sont au pluriel: acquiescements, arrivages, conditions, explications, odeurs, protestations, sanctions. Il est vrai que très souvent la marque du pluriel n'est pas repérable à l'oreille. Mais vu le nombre d'occurrences, nous concluons que le pluriel est licite dans cette position. Dès lors que les noms déverbaux au pluriel expriment souvent le résultat concret de l'action, par exemple confessions (au pluriel) comme résultat de l'acte de (se) confesser, la présence du pluriel confirme l'observation selon laquelle rien n'empêche que les noms soient concrets.

\subsection{La pronominalisation}

La pronominalisation du nom nu est possible, comme le montre Naganuma cité en (7). 
(7) Nous avions déjà l'art d'opinion, la démocratie d'opinion; nous aurons maintenant l'enseignement d'opinion. Il faut renoncer à l'idée que le lycée puisse rester un lieu privilégié de communication du savoir.

Conclusions? Elles sont au nombre de deux. La première était déjà contenue dans mes prémisses.

(Le Nouvel Observateur, 1772, 22 octobre 1998, cité par Naganuma 2004)

Cette caractéristique suggère, avec l'emploi de la forme au pluriel, que le nom nu dans cette construction garde pleinement sa nature substantive ; il est référentiel et n'a pas de valeur attributive.

\subsection{L'interrogation et la négation}

La phrase nominale avec un nom nu sans déterminant peut être interrogative comme le montre l'exemple (8).

(8) Tout cela est bien beau, mais va-t-on enfin dénicher de la vie ? Certainement pas de petits hommes verts, mais quelque chose comme des bactéries-traces, comme on a cru en retrouver l'an dernier sur une météorite très spéciale (lire ci-contre) ?

Preuve que de la vie a peut-être émergé sur la planète voilà plus de 3 ou 4 milliards d'années ? Franchement, il faut dire que, pour cette fois, ce devrait être non. Non par malchance.

(Libération, Par Dominique Leglu, 4 juillet 1997, $<$ http://www.liberation.fr/sciences/1997/07/04/ce-soir-rendez-vous-sur-mars-lerobot-rocky-arrivera-t-il-indemne-sur-mars-1-engin-americain-muni-d-_210881>, consulté le 15 mars 2018)

Elle peut être négative, même si le cas est rare.

(9) Le forum «Ambiance et conditions de travail » du site internet Tchooze.com ressemble un peu à la place de la Nation, à Paris, un jour de manifestation. Mais ici ni banderoles ni pétards. Juste des tracts virtuels déposés par des travailleurs en colère. Parmi eux, de plus en plus de start-uppeurs, ces jeunes salariés de la nouvelle économie, exaspérés par leurs conditions de travail

(Le Nouvel Observateur, $1^{\mathrm{er}}-7$ février 2001, cité par Lefeuvre 2005)

Nous verrons plus tard que certaines phrases nominales avec un nom nu ont une fonction présentative ( $c f .6 .3 .2)$. Pourtant, ces deux caractères suggèrent qu'elle n'équivaut pas à un présentatif pur, comme la construction voilà, qui ne peut être ni interrogative, ni négative.

\subsection{Les qualificatifs}

Souvent, dans la phrase nominale, le nom nu en tête est suivi d'une proposition qualificative. Dans l'immense majorité des cas, le pronom relatif employé est qui, comme en (1), (2) et, infra, (17), (18), (20), (22) (26) et (27). Il est difficile de trouver des exemples avec d'autres relatifs - nous n'en avons pas relevé avec que (dans l'exemple (8) que n'est pas un pronom relatif, mais un conjonctif.) L'exemple (10) est un cas rare de phrase nominale avec auxquelles.

(10) Mais cette pétition de principe implique des conséquences prosaïques auxquelles le musée ne peut que se soumettre. En effet, la photographie, en se plaçant ainsi “dans l'antique temple du Beau", en adopte les valeurs fondamentales d'unicité 
et de rareté. Valeurs philosophiques auxquelles on croyait la photographie totalement étrangère mais aussi valeur marchande sur laquelle se fonde toute la stratégie commerciale qui nous environne et que nous ne pouvons ignorer.

(Alain Sayag, Préface de : Paris des photographes, 1997)

En (10), le nom nu valeurs, présent dans la phrase précédente, réapparaît à la ligne suivante, devenant le leitmotiv de la séquence.

Or, avec qui, le nom nu fonctionne comme sujet et la proposition relative commence par un syntagme prédicatif, c'est-à-dire un ordre qui correspond à celui de la prédication standard. Les occurrences très fréquentes de qui rappellent qu'une des structures typiques de la phrase nominale avec un nom nu est « thème - rhème ».

\subsection{L'insertion d'un élément en position frontale}

Le nom nu occupe presque toujours la tête de la phrase nominale. L'insertion d'un élément en position frontale est difficile, le paradigme s'avérant très limité. On compte quelques expressions de localisation spatio-temporelle, comme en (11) où hier se situe devant confessions et en (12) où ici apparaît devant nouvelles complications.

(11) Je suis sûr que mon malheureux ami ne mérite pas le nom de mauvais prêtre. Je suppose même qu'il est sincèrement attaché à sa compagne, car je l'ai connu jadis sentimental. Le prêtre médiocre, hélas ! l'est presque toujours. Peut-être le vice est-il moins dangereux pour nous qu'une certaine fadeur? Il y a des ramollissements du cerveau. Le ramollissement du cœur est pire.

En revenant ce matin de mon annexe, à travers champs, j'ai aperçu M. le comte qui faisait quêter ses chiens le long du bois de Linières. Il m'a salué de loin, mais ne semblait pas très désireux de me parler. Je pense que d'une manière ou d'une autre il a connu la démarche de Mademoiselle. Je dois agir avec beaucoup de réserve, de prudence.

Hier, confessions. De trois à cinq, les enfants. J'ai commencé par les garçons, naturellement.

Que Notre-Seigneur les aime, ces petits ! Tout autre qu'un prêtre, à ma place, sommeillerait à leur monotone ronron qui ressemble trop souvent à la simple récitation de phrases choisies dans l'Examen de conscience, et rabâchées chaque fois...

(Georges Bernanos, Journal d'un curé de campagne, cité par Lefeuvre 2000)

\section{LAPINS DE GARENNE ET LAPINS DE CHOUX}

Le décret de Bordeaux sur l'organisation des gardes nationales mit fin à cette situation intolérable. Au souffle puissant des triumvirs, prrrt ! les plumes de coqs s'envolèrent, et tous les francs-tireurs de Tarascon - chacals, espingoliers et autres - vinrent se fondre en un bataillon d'honnêtes miliciens, sous les ordres du brave général Bravida, ancien capitaine d'habillement.

Ici, nouvelles complications. Le décret de Bordeaux faisait, comme on sait, deux catégories dans la garde nationale : les gardes nationaux de marche et les gardes nationaux sédentaires ; «lapins de garenne et lapins de choux », disait assez drôlement le receveur Pégoulade.

(Alphonse Daudet, Contes Du Lundi, cité par Lefeuve 2000)

En (11) et (12), les noms nus introduisent un nouvel élément dans le texte, en dehors du cotexte antérieur. Il s'agit d'informations nouvelles. C'est la structure informationnelle de la phrase nominale qui rend licite l'insertion. Les expressions de localisation spatio- 
temporelle hier et ici viennent atténuer l'impression de rupture que provoque l'intrusion d'un élément inattendu en reliant la phrase à ce qui la précède.

Un autre élément peut s'intercaler avant le nom nu : la conjonction mais. Dans le texte cité en (13), l'insertion est admissible devant Peines perdues.

(13) S'était-il couché sous le lierre, ou dans quelque trou ? On chercha durant deux semaines. On fit venir de Paris un jeune policier, ambitieux et tenace, Gorgeret, qui avait déjà réussi des coups de maître. Peines perdues. Investigations sans résultat. L'affaire fut classée, au grand ennui de Gorgeret, qui se promit bien de ne jamais l'abandonner.

(Maurice Leblanc, La femme aux deux sourires)

Dans (9), que nous reproduisons comme (14), nous observons le cumul de la conjonction mais et de l'élément locatif ici.

(14) Le forum «Ambiance et conditions de travail » du site internet Tchooze.com ressemble un peu à la place de la Nation, à Paris, un jour de manifestation. Mais ici ni banderoles ni pétards. Juste des tracts virtuels déposés par des travailleurs en colère. Parmi eux, de plus en plus de start-uppeurs, ces jeunes salariés de la nouvelle économie, exaspérés par leurs conditions de travail

(Le Nouvel Observateur, $1^{\mathrm{er}}-7$ février 2001, cité par Lefeuvre 2005)

Dans l'exemple (14), si la phrase commençait par ni banderoles, ni pétards, sans la conjonction mais, l'enchaînement textuel laisserait croire que ces éléments n'apparaissent pas non plus lors des manifestations. De plus, l'élément locatif ici, que l'on pourrait remplacer par cette fois, renforce la liaison. Bref, ce qui est inséré en position frontale contribue à relier la phrase nominale au cotexte antérieur.

On peut aussi insérer l'adjectif autre, comme le montre (15).

Enfin, pour compliquer encore l'établissement d'une relation dose-effet, ces contaminants sont présents à faibles doses pour la population générale. La concentration en dioxines est de l'ordre du nanogramme $\left(1 \mathrm{ng}=10^{-9} \mathrm{~g}\right)$ par mètre cube d'air (cent fois plus pour les vieux incinérateurs), et du picogramme (1 pg $=10^{-12} \mathrm{~g}$ ) par gramme de matière grasse (abrégé en $\mathrm{MG}$ ) dans les aliments.

Autre fait de nature à renforcer les incertitudes : la dioxine de Seveso est peu (ou pas du tout) détectée dans les aliments sur lesquels on crie haro...

(Science \& Vie, $\mathrm{n}^{\circ}$ 968, mai 1998)

L'effacement d'autre rendrait l'enchaînement incongru. Fait et son complément ne renvoient pas au cotexte antérieur; ils livrent une information nouvelle dont le contenu apparaît après un deux-points.

Il en va de même pour l'exemple (16), où tout comme (15) la phrase nominale qui commence par Autre fait se trouve au début d'un paragraphe.

Une chose est certaine : c'est que, sans être encore - tant s'en faut - légion, de plus en plus nombreux se retrouvent, d'une occasion de rencontre à l'autre, ceux qui n'hésitent plus aujourd'hui à se déclarer ouvertement "guillaumiens" et que d'année en année ne cesse de croître le nombre des écrits et essais inspirés par les vues et théories de Gustave Guillaume. Tant et si bien que l'on parle volontiers désormais, extra muros, d'une école guillaumienne de linguistique et que l'auteur d'un ouvrage de vulgarisation récemment consacré à cette école dans une collection largement répandue a pu, le printemps dernier, organiser avec un franc succès, à la Vrije Universiteit Brussel où il enseigne, un colloque international où psychomécaniciens du langage et générativistes ont eu 
l'occasion de confronter, sur des thèmes établis d'un commun accord, la puissance explicative de leur méthode respective.

Autre fait significatif: la rapidité relative avec laquelle s'enlèvent, depuis dix ans, les écrits divers - rééditions ou inédits - de Gustave Guillaume. On le lit. On l'étudie. Il inspire des thèses d'année en année plus nombreuses. On s'adresse au Fonds Gustave Guillaume, où sont conservés ses manuscrits, pour obtenir photocopie des textes disponibles sous forme de dactylogrammes en attendant publication. Les temps semblent lointains où, dans telle université française, un candidat au doctorat se faisait répondre par son patron: "Guillaume? Connais pas". Ou encore mieux: "Ce prétendu linguiste ne sait même pas ce que c'est qu'un fait linguistique". C'était pourtant hier.

(Roch Valin, Préface de : Le problème de l'article et sa solution dans la langue française)

Dans Autre fait significatif, la présence de l'adjectif Autre est obligatoire. Sémantiquement, il crée à la fois une opposition et un lien. Une opposition en tant qu'il marque le changement de fait. Un lien parce qu'il présuppose un élément identique dans le cotexte antérieur. Dans cette phrase nominale, le nom nu ne pourrait seul assurer la cohésion.

\section{La relation au cotexte antérieur}

Nous distinguons trois modalités de relation du nom nu en phrase nominale avec le cotexte antérieur : (i) la répétition, (ii) la reprise hyperonymique et (iii) les autres cas.

\subsection{La répétition}

Assez souvent, le nom nu utilisé en phrase nominale est une itération du cotexte, comme en (17) thème, en (18) conflit et diversité, métier et personnalités en (19).

(17) À travers la métaphore oiseau-poisson, c'est donc un thème beaucoup plus vaste qui se propose, celui de la réversibilité de l'univers et de l'existence. Thème familier à l'imagination baroque, qui s'est ingéniée à transposer dans sa littérature les jeux de perspective et les mirages en trompe-l'œil chers à l'architecture et à la peinture de cette époque.

(Gérard Genette, « Un univers réversible » Figures I, 1966)

(18) Un conflit acharné se poursuit à Kyoto entre le maire et les bonzes sur la mise en application d'une taxe perçue pour assurer la préservation des sites historiques et religieux de l'ancienne capitale japonaise. Conflit qui met en jeu la « liberté du culte », prétendent les responsables de l'Association des bouddhistes de Kyoto. Cependant, depuis quelque temps, on commence à soupçonner que la « liberté du culte » seule n'est pas en cause. On vient de découvrir, en effet, la présence d'un certain agent immobilier qui tire les ficelles derrière la direction de l'organisme bouddhiste.

(Journal Asahi, ${ }^{\text {er }}$ février 1986, Traduit par d'Ohga 1989)

(19) Car ce qui ressort, d'un examen, fût-il superficiel, c'est l'extraordinaire diversité de la création à Paris. Diversité due à des apports successifs et multiples. Cela tient d'abord à la relative marginalité du métier de photographe. Métier alors encore à peine codifié mais en pleine expansion et qui de ce fait attire de fortes personnalités dont l'origine sociale ou nationale aurait freiné l'ascension dans d'autres domaines mieux "balisés". Personnalités souvent riches de talents divers comme le hongrois Brassaï, l'américain Man Ray, l'allemand Bellmer, 
parfois même extravagants comme Claude Cahun ou le roumain Eli Lotar qui trouvèrent dans la photographie à la fois un moyen de vivre (ou plutôt de survivre), une grande liberté et finalement un moyen de s'accomplir. Diversité aussi des pratiques (professionnelles ou artistiques) diversité enfin des esthétiques qui s'inscrivent avec plus ou moins de bonheur dans les grands courants artistiques qui scandent le siècle.

(Alain Sayag, Préface de : Paris des photographes, 1997)

Dans l'exemple (2), juste avant la première phrase nominale, le mot couvercle figure dans le texte.

En (20), une partie du syntagme nominal vacances est apparue dans le cotexte antérieur.

(20) Un nombre croissant de Japonais passent leurs vacances de fin d'année et du nouvel an à l'étranger. Forme de vacances qui s'insère de plus en plus dans leur mode de vie.

(Journal Asahi, 20 novembre 1987, Traduit par d'Ohga 1989)

L'exemple (21) n'est pas prototypique en ce sens que la lettre initiale du nom nu n'est pas en majuscule, bien qu'on puisse très bien le réécrire en remplaçant le deux-points qui le précède par un point.

(21) En effaçant, autant que faire se peut, de cette gradation toute connotation valorisante, on pourrait situer dans la région moyenne un type de récit trop éloigné des poncifs du vraisemblable pour se reposer sur le consensus de l'opinion vulgaire, mais en même temps trop attaché à l'assentiment de cette opinion pour lui imposer sans commentaire des actions dont la raison risquerait alors de lui échapper: récit trop original (peut-être trop «vrai ») pour être encore transparent à son public, mais encore trop timide, ou trop complaisant, pour assumer son opacité.

(Gérard Genette, « Vraisemblance et motivation », Figures II, 1969)

Le nom nu récit est mis après le deux-points qui est utilisé pour introduire diverses catégories de segments - une explication, une conséquence ou une synthèse. De fait, la phrase nominale à nom nu fonctionne souvent sur ce registre.

En cas de répétition du nom, la cohésion lexicale est explicite. Le nom nu répété est en règle générale accompagné de termes modificatifs, tels qu'un adjectif qualificatif ou une proposition relative, qui représente la partie rhématique de la phrase. Sans cet ajout, il n'y aurait pas de progression textuelle. Comme nous l'avons évoqué avec l'exemple (10), la phrase nominale peut faire progresser le texte tout en se structurant «thème-rhème ». En effet, l'expression répétée dans la position frontale d'une phrase est typique d'un thème.

\subsection{La reprise par un hyperonyme}

L'exemple (22) illustre la reprise par un hyperonyme.

À côté des journalistes moraux, sous cette pluie d'homélies comme sous une pluie d'été dans quelque parc, il a surgi, entre les planches du tréteau saintsimonien, une théorie de petits champignons d'une nouvelle espèce assez curieuse, dont nous allons faire l'histoire naturelle.

Ce sont les critiques utilitaires. Pauvres gens qui avaient le nez court à ne le pouvoir chausser de lunettes, et cependant n'y voyaient pas aussi loin que leur nez.

(Théophile Gautier, Mademoiselle de Maupin) 
En l'espèce, Pauvres gens reprend l'expression les critiques utilitaires, en y adjoignant une appréciation défavorable. Dans la reprise hyperonymique, il est fréquent, comme dans cet exemple, que le nom nu porte un jugement ou introduise une nouvelle perspective sur les informations précédentes en les classant ou en les commentant. Le groupe nominal est en ce sens rhématique.

Dans l'exemple (23), sape précède le nom nu lieu. Et l'adjectif qualificatif formidable le commente.

(23) Au-dessous de toutes ces mines que nous venons d'indiquer, au-dessous de toutes ces galeries, au-dessous de tout cet immense système veineux souterrain du progrès et de l'utopie, bien plus avant dans la terre, plus bas que Marat, plus bas que Babeuf, plus bas, beaucoup plus bas, et sans relation aucune avec les étages supérieurs, il y a la dernière sape. Lieu formidable. C'est ce que nous avons nommé le troisième dessous. C'est la fosse des ténèbres. C'est la cave des aveugles. Inferi.

(Victor Hugo, Les Misérables Tome III (1890), cité par Naganuma 2004)

Dans cette position, l'utilisation d'un hyponyme semblerait moins naturelle. Dans le texte cité en (22), si l'ordre d'apparition des deux expressions est inversé, comme en (24), l'enchaînement paraît moins naturel, voire erroné.

(24) \# Ce sont les Pauvres gens. Critiques utilitaires qui avaient le nez court à ne le pouvoir chausser de lunettes, et cependant n'y voyaient pas aussi loin que leur nez.

Il arrive que la reprise soit réalisée par un pantonyme, $i$. e. un terme à forte généricité hyperonymique, un mot passe-partout, tel que truc, machin ou chose. C'est le cas en (25) : le groupe nominal Toutes choses renvoie aux éléments soulignés dans le cotexte antérieur.

(25) Il faut imaginer le choc culturel que représente pour le groupe japonais le choix de l'Hexagone avec ses lois sociales favorables aux salariés : les 39 et bientôt 35 heures de travail hebdomadaires, les 5 semaines de congés payés, des syndicats capables de paralyser le pays, etc. Toutes choses évidemment inconnues au Japon mais aussi en Grande-Bretagne où la protection sociale est moindre. "Pour Toyota, c'est vraiment un choix révolutionnaire, car la France traîne une mauvaise image à l'étranger », dit un responsable français.

(Libération, Par Frédérique Amaoua, 4 décembre 1997 $<$ http://www.liberation.fr/futurs/1997/12/04/dans-le-nord-y-aura-toyota-1arrivee-du-constructeur-japonais-a-valenciennes-va-creer-1500emplois_223588>, consulté le 13 mars 2018)

La qualification est exploitée pour compenser l'indécision anaphorique du pantonyme. En (25), choses est précisé par des éléments adjectivaux qui relaient la progression textuelle.

\subsection{Les autres cas}

Le nom nu dans la phrase nominale n'étant pas lié au cotexte antérieur par le déterminant, la cohésion lexicale peut être assurée par la répétition littérale ou hyperonymique. Sinon, la relation sera d'ordre strictement lexical, avec, le plus souvent, l'ajout d'une information nouvelle. Nous distinguerons trois cas : (i) l'enchaînement rétrospectif, (ii) l'enchaînement présentatif et (iii) l'enchaînement prospectif. (26) contient un exemple de chaque sorte.

(26) Ils [= les énoncés (1)-(4)] sont acceptés sans réserve par les sujets parlants, qui les trouvent très proches par le sens. Si de ce point de vue on leur demande 
cependant de comparer (1) à (3) et (2) à (4), la réponse unanime est que la différence essentielle réside en ce que dans (4), et à un degré moindre dans (3), on « voit » l'allusion en train d'être faite. Glose qui ressemble à s'y méprendre à celle faite à propos de l'imparfait. Autre exemple surprenant, trouvé dans un périodique peu coutumier des fleurs de style : «(..) aux angles des rues, des Latino-Américains baragouinant à peine l'anglais vendent, contre dollars, des journaux en cyrillique à de vieilles "babouchkas" serrées dans leurs fichus..." (Le Monde, 21/11/85, p. 1 : c'est nous qui soulignons). Là encore, les sujets parlants déclarent, à l'audition de cet énoncé, "voir» les dollars changer de mains. Intuition qui recoupe l'analyse que donnent les grammaires traditionnelles de certains cas d'omission de l'article : "dans une énumération, pour donner à celle-ci un caractère plus vif» (Wartburg et Zumthor, p. 295); «dans certaines énumérations, pour la vivacité de l'expression» (Grevisse, p 361).

(Jean-Claude Anscombre, "L’article zéro en français : un imparfait du substantif?", 1986)

(i) L'enchaînement rétrospectif

Glose synthétise la dernière partie de la phrase précédente. Le texte est rétrospectif : il commente le cotexte antérieur. Une paraphrase de cette phrase nominale serait: C'est une glose...

(ii) L'enchaînement présentatif

La phrase nominale qui commence par Autre exemple se comporte comme une construction présentative. En effet, nous pouvons la paraphraser par Voici un autre exemple... ou Il y a un autre exemple... Elle introduit dans le texte un élément nouveau. Le contenu de l'« exemple » est décrit ultérieurement après un deux-points. La phrase ne revient pas sur le cotexte antérieur ; son rôle est rhématique avec l'adjonction d'une nouvelle information.

(iii) L'enchaînement prospectif

Dans la troisième phrase, le contenu d'intuition est complété par la citation. Si cette forme est identique au type précédent (ii) (Autre exemple...), la structure informationnelle ne l'est pas. Pour intuition, il existe, dans la phrase précédente, l'expression les sujets parlants qui l'anticipe. Ce type s'apparente au cas de la reprise hyperonymique (cf. 6.2) : le nom nu en position frontale a pour référence un item lexical qui le suggère dans le cotexte antérieur. Ainsi le nom nu peut fonctionner comme un thème et structurer la phrase nominale en "thème-rhème ». Le nom nu ne commente pas le cotexte précédent, l'ensemble de la phrase étant orientée vers le cotexte qui suit en participant à la progression du texte. On la qualifie donc de prospective.

Voici d'autres exemples de ces trois enchaînements.

\subsubsection{L'enchaînement rétrospectif}

Comme exemple d'enchaînement rétrospectif, prenons l'exemple (27) : le nom nu Vérité synthétise ce qui vient d'être relaté.

(27) Tout le volume incite à la réflexion profonde. Il fait voir une fois de plus que l'instinct populaire, en puissance et en aisance d'abstraction, peut l'emporter sur la pensée consciente. Vérité paradoxale, mais qui se constate souvent en grammaire.

(Louis Havet, Journal des 1919, $<$ https://archive.org/stream/journaldessavant1919acaduoft/journaldessavant1919 acaduoft_djvu.txt>, consulté le 9 octobre 2017) 
Cet exemple rejoint (1) où le nom nu résumait une partie du cotexte antérieur. Le contenu sémantique des noms n'est pas intrinsèquement connecté au cotexte. Ils endossent cependant une fonction rétrospective en introduisant un commentaire de l'information précédente, souvent accompagné d'un qualificatif exprimant un jugement (paradoxale en (27)) ou une impression (comique en (1)). Ce type d'enchaînement, dont le groupe nominal est rhématique s'apparente au cas de la reprise hyperonymique (cf. 6.2), où le nom nu donne un jugement portant sur le cotexte antérieur.

Ce fait est surtout observable avec le nom exprimant une appréciation, comme dans l'exemple suivant.

(28) Profitez-en car ce service, l'un des plus efficaces selon les habituées de Montreuil, devrait disparaître prochainement.

Dommage, à l'heure où l'Urssaf de Paris vit une petite révolution. Sous la houlette d'une nouvelle directrice, Suzanne Belz, la tour de Montreuil est un train de rompre avec vingt ans d'organisation taylorienne du travail.

(Le Nouvel Observateur, 1775, 12 novembre 1998, cité par Naganuma 2004)

Il est vrai que Dommage dans cet exemple peut être considéré comme une forme elliptique de la construction "C'est dommage ». Mais, du point de vue fonctionnel, nous ne voyons pas la nécessité de distinguer ce vocable d'autres noms nus placés dans la même position.

\subsubsection{L'enchaînement présentatif}

Les phrases nominales à enchaînement présentatif sont, dans nos données, les plus nombreuses. Elles sont souvent très courtes comme l'illustre l'exemple (29). Le groupe nominal Lacune effrayante annonce une information nouvelle.

(29) La personnalité semble au moins aussi énergique dans la chenille vigoureuse que dans le papillon si mou. Donc, c'est bien réellement son être personnel qu'elle laisse courageusement sécher, s'anéantir, pour devenir quoi ? rien de rassurant, une courte masse molle, blanchâtre. Ouvrez la nymphe peu après qu'elle a filé ; dans son linceul vous ne trouvez qu'une sorte de fluide laiteux, où rien n'apparaît, à peine de douteux linéaments qu'on voit ou que l'on croit voir. Dans quelque temps, vous pourrez, avec une fine aiguille, isoler ces je ne sais quoi, et vous figurer que ce sont les membres du futur papillon. Lacune effrayante. Il y a (pour beaucoup d'espèces) un moment où rien de l'ancien ne parait plus, où rien du nouveau ne paraît encore.

(Jules Michelet, L'insecte)

Les noms nus en (30), en (31) et en (32) apportent aussi des informations nouvelles, lexicalement très peu prévisibles en regard du cotexte antérieur.

(30) Les talibans [...] avaient accueilli chez eux un opposant saoudien [...] qui s'empressa de commanditer et d'organiser deux attentats contre des ambassades américaines en Afrique de l'Est, tuant plus de 200 personnes. Fureur des ÉtatsUnis, qui bombardent des camps d'entraînement de terroristes en Afghanistan et réclament Ben Laden. Refus du mollah Omar. Sanctions décidées par l'ONU (Le Nouvel Observateur, 12-18 avril 2001, cité par Lefeuvre 2005)

(31) Souvenez-vous. Le 9 juin dernier, Robert Lagier, 65 ans, un maçon pied-noir devenu colleur d'affiches du Front national, comparaissait avec deux complices devant la cour d'assises d'Aix-en-Provence pour avoir tué, d'une balle dans le dos, Ibrahim Ali, un lycéen français d'origine comorienne. Rien à voir avec le racisme ou la xénophobie, se défend-il, "c'était un accident». Il s'est senti 
agressé, il a tiré par peur, répète inlassablement son avocat. Jusqu'au jour où, le 15 juin, Julie vient dire aux jurés que son grand-père n'est pas l'homme qu'il prétend : «Il m'emmenait au club de tir pour m'apprendre à tirer sur les Arabes, les "melons", comme il les appelait. J'avais 8 ans à l'époque. » Silence suffoqué de l'assistance. Assis dans son box, Robert Lagier accuse le coup, livide. Une semaine plus tard, le verdict tombe : deux ans de prison pour Pierre Giglio, dix ans pour Mario D’Ambrosio, quinze ans pour Robert Lagier, malgré le cancer généralisé dont il est atteint.

(L'Express, 2463, 17 septembre 1998, cité partiellement par Naganuma 2004)

Dans le Nord, y'aura Toyota.

L'arrivée du constructeur japonais à Valenciennes va créer 1500 emplois.

Il n'y a pas si longtemps, on aurait crié au loup ! Aujourd'hui, le gouvernement français s'apprête à dérouler le tapis rouge et ouvrir grandes les portes de Matignon à Toyota pour que le premier constructeur automobile japonais y fasse l'annonce de sa décision de s'implanter en France. Mieux, dans la ville sinistrée de Valenciennes. Changement d'époque. Et d'analyse. «De toute façon, ils se seraient installés en Europe et auraient inondé de la même manière le marché français, dit un haut fonctionnaire français proche du dossier. Mieux vaut qu'on en récupère l'investissement et les emplois. »

(Libération, Par Frédérique Amaoua, 4 décembre

1997 $<$ http://www.liberation.fr/futurs/1997/12/04/dans-le-nord-y-aura-toyota-1arrivee-du-constructeur-japonais-a-valenciennes-va-creer-1500emplois_223588>, consulté le 13 mars 2018)

En (29), (30), (31) et (32), la phrase nominale actualise ; elle n'est ni rétrospective ni prospective. Elle ne requiert aucun élément qui la connecterait au cotexte environnant. Elle peut être courte, sans développement de qualificatifs. En effet, seules s'inscrivent dans cette catégorie, semble-t-il, les holophrases nominales telles que Résultat en (2), Problème en (3), Explication en (4) et Conclusions en (7). Voici d'autres noms attestés dans cette configuration minimale : erreur, motif, paradoxe, protestations, résumé, sérénité, trahison.

Rappelons la possibilité de l'insertion d'un élément devant le nom nu (cf. 5.5). Cette insertion, assez restreinte d'ailleurs, ne semble possible qu'avec l'enchaînement présentatif. Dans l'exemple suivant fugue est précédé de nouvelle.

Abdelkader, Béglais de 15 ans, est un prototype du mineur à la dérive. Depuis l'âge de 13 ans, il a multiplié les infractions et les délits. La justice, engorgée, a mis du temps à y répondre. Déstabilisé, orphelin de père, il n'est pas, selon son avocat, un "caïd" qui agit par bravade. Placé dans un foyer, il s'en est enfui il y a deux mois en volant une voiture du centre après un conflit avec un éducateur. Alors le juge des enfants a décidé de le placer dans le flambant neuf $\mathrm{CEF}$ de Sainte-Eulalie. Pendant quinze jours il se tient à carreau. Mais quand le juge le condamne à du sursis et à 6 mois de mise à l'épreuve, il ne supporte pas. Nouvelle fugue.

Désordre : les centres fermés, principale innovation du gouvernement Raffarin, se devaient d'être des sanctuaires de discipline. Abdelkader est recherché, le ministre de la Justice affirme qu'il doit aller en prison. A peine retrouvé chez sa mère, Abdelkader a donc été - selon le vœu de Dominique Perben - écroué à la maison d'arrêt de Rennes. Ainsi la fugue, traditionnelle chez ces jeunes, devient-elle quasiment une évasion dans ce dispositif, qui ne laisse pas de deuxième chance.

(Le Nouvel Observateur, 26 juin $2003<\mathrm{http}$ //citizenmorue.free.fr/M-Files/MFiles-6.htm>, consulté le 11 mars 2018, cité partiellement par Lefeuvre 2005) 
Reprenons aussi l'exemple (26), dont nous reproduisons ici le début comme (34).

(34) Ils [= les énoncés (1)-(4)] sont acceptés sans réserve par les sujets parlants, qui les trouvent très proches par le sens. Si de ce point de vue on leur demande cependant de comparer (1) à (3) et (2) à (4), la réponse unanime est que la différence essentielle réside en ce que dans (4), et à un degré moindre dans (3), on « voit » l'allusion en train d'être faite. Glose qui ressemble à s'y méprendre à celle faite à propos de l'imparfait. Autre exemple surprenant, trouvé dans un périodique peu coutumier des fleurs de style: «(..) aux angles des rues, des Latino-Américains baragouinant à peine l'anglais vendent, contre dollars, des journaux en cyrillique à de vieilles "babouchkas" serrées dans leurs fichus... " (Le Monde, 21/11/85, p. 1 : c'est nous qui soulignons).

Dans Autre exemple, la présence de l'adjectif Autre est obligatoire. Il inscrit cette phrase nominale dans une série, tout en indiquant une rupture, un changement. Cet exemple (34) offre la structure typique d'un enchaînement présentatif. Au demeurant, tous les exemples incluant un élément inséré se classent dans cette catégorie. (Cf. (11), (12), (13), (14), (15) et (16)). Au moment de présenter un élément nouveau, l'énoncé s'accroît, en position frontale, d'items qui font le lien avec le cotexte antérieur.

À la différence du cas de la répétition ( $c f .6 .1)$, dans l'enchaînement présentatif le thème est absent. Cela explique la possibilité, voire la nécessité de l'insertion d'un élément devant le nom nu (Autre en (15) (16) et (34)). Dépourvu de thème, le nom nu a parfois besoin d'être relié au cotexte antérieur au moyen d'un élément explicite.

\subsubsection{L'enchaînement prospectif}

Dans certains exemples d'enchaînement prospectif impliquant un élément apparu dans le cotexte antérieur, le nom nu entretient une relation qui s'apparente à l'anaphore associative. En (35), livre a été préfiguré, dans la phrase précédente, par des notions qui lui sont étroitement liées : lecture et pages.

(35) Je n'ai pu soutenir cette lecture, non par émotion, n'en étant pas encore émue, mais par dégoût de l'horrible que j'ai senti dès l'abord aux premières pages. Livre fermé. Ce n'était pas ce qu'il fallait à ma disposition d'âme ; je m'étais trompée en cherchant un poids, tandis qu'il faut s'alléger alors.

(Eugénie de Guérin, Eugénie de Guérin: journal et fragments $<$ http://gallica.bnf.fr/ark:/12148/bpt6k202673b/texteBrut>, consulté le 23 octobre 2017, cité par Naganuma 2004)

Si livre apporte une information nouvelle, il n'est pas complètement inattendu. Il a été rendu lexicalement prévisible par le cotexte antérieur. C'est par là que ce type d'enchaînement se distingue de l'enchaînement présentatif. Il est différent de l'enchaînement rétrospectif aussi : la phrase nominale ne commente pas le cotexte antérieur. En ce qui concerne la relation entre le nom nu et le cotexte antérieur, ce type s'apparente au cas de la reprise hyperonymique ( $c f .6 .2)$. Or, la structure informationnelle de l'ensemble de la phrase rappelle plutôt la répétition du même nom $(c f .6 .1)$ : la phrase nominale fait progresser le texte tout en se structurant « thème-rhème ».

Prenons un autre exemple :

(36) L'un des premiers livres faisant clairement état d'un assassinat fut écrit en 1964 par un ancien du FBI. Une sorte de Fox Mulder de drôles d'X Files, Frank Cappell, qui s'interrogeait en 70 pages sur L'Etrange Mort de Marilyn Monroe (Strange Death of Marilyn Monroe). Opuscule flingué à mort dès parution. Moqué. Mais lu de près par le tsar du renseignement, le dictateur de la flicaille, 
Edgar J. Hoover, directeur inamovible et haï du FBI. Lu également à la loupe par toute une famille du Massachusetts qui venait de perdre, en novembre 1963, un de ses fils en la personne d'un président des Etats-Unis. Et ce n'était pas fini. (L'Express «La vérité sur un crime d'Etat», 15 octobre 1998, $<$ https://www.lexpress.fr/culture/cinema/la-verite-sur-un-crime-d-

etat_495032.html>, consulté le 19 novembre 2017, cité partiellement par Naganuma 2004)

Si le nom nu opuscule lui-même n'est pas mentionné dans le cotexte antérieur, son synonyme livres est déjà présent. Il peut donc facilement se présenter comme le thème majeur de la séquence. En effet, les qualificatifs suivant ce nom nu apportent une information nouvelle pour constituer la partie rhématique. Comme le signale Naganuma (2004), on peut paraphraser cette phrase nominale par «L'opuscule fut flingué à mort dès parution. »

Nous avons peu d'exemples de ce type d'enchaînement. Cette relative rareté pourrait s'expliquer par la difficulté que présente la mise en relation avec le cotexte antérieur.

\section{Conclusion}

Nous avons proposé cette analyse de la phrase nominale avec un nom nu afin d'examiner un cas de cohésion exclusivement lexicale. Nous avons montré que, si les noms nus dans la phrase nominale sont le plus souvent abstraits, ils peuvent être concrets et qu'ils conservent pleinement leur nature substantive. Parmi les relations qu'entretient ce nom nu avec le cotexte antérieur, nous avons constaté deux cas prépondérants : la répétition et la reprise hyperonymique. Pour les autres cas, nous avons été amenée à mettre l'accent sur la structure informationnelle en distinguant entre les enchaînements rétrospectif, présentatif et prospectif. Dans le cas de la répétition et celui de l'enchaînement prospectif, le nom nu est thématique, alors que dans les autres trois cas, le groupe nominal est rhématique. L'enchaînement présentatif se singularise de trois façons. (i) Le nom nu actualise un élément nouveau. (ii) La phrase est souvent courte. (iii) L'insertion d'un élément devant le nom nu est possible.

Un examen des autres cas d'absence de déterminant permettra de compléter cette analyse.

* La présente recherche bénéficie de l'Aide à la recherche scientifique (C) de KAKENHI (15K02522), accordée par la Société Japonaise pour la Promotion des Sciences (JSPS).

\section{Références bibliographiques}

Adam J.-M. (1999). Linguistique textuelle, des genres de discours aux textes. Paris : Nathan.

Adam, J.-M. (2011). La linguistique textuelle. Introduction à l'analyse textuelle des discours. Paris : Armand Colin.

Adam, J.-M. (2017). Les textes, types et prototypes. Paris : Armand Colin.

Adam, J.-M. (2018). Le paragraphe : entre phrases et texte. Paris : Armand Colin.

Anscombre, J.-C. (1986a). L'article zéro en français : un imparfait du substantif? Langue française, 72, 4-39.

Anscombre, J.-C. (1986b). L'article zéro, termes de masse et représentation d'événements en français contemporain. Recherches linguistiques, XI, 5-34.

Anscombre, J.-C. (1991a). La détermination zéro : quelques propriétés. Langages, 102, 103-124. 
Anscombre, J.-C. (1991b). L’article zéro sous préposition. Langue française, 91, 24-39.

Bally, C. (1965). Le langage et la vie. Genève : Droz.

Benetti, L. (2008). L'article zéro en français contemporain: aspects syntaxiques et sémantiques. Berne : Peter Lang.

Buvet, P.-A. (2009). Détermination prédicative et article zéro. Synergies Tunisie, 1, 145-158.

Charolles, M. (1994). Cohésion, cohérence et pertinence du discours. Travaux de linguistique, 29, $125-151$.

Charolles, M. (2006). Cohérence, pertinence et intégration conceptuelle. Des discours aux textes : modèles et analyses. in Lane, P. (éd) Rouen, Presses Universitaires de Rouen : 39-74.

Dobrovie-Sorin, C. (2005). Noms nus et généricité, Saint-Denis : Presses Universitaires de Vincennes.

Furukawa, N. (1978). Article zéro ou absence d'article? Bulletin d'études de linguistique française, $12,35-46$.

Furukawa, N. (1986). L'article et le problème de la référence en français. Tokyo : Furansu Tosho.

Giry-Schneider, J. (1991). L'article zéro dans le lexique-grammaire des noms prédicatifs. Langages, $102,23-35$.

Grevisse, M. et Goosse, A. (2016). Le bon usage : grammaire française. Louvain-la-Neuve : De Boeck Supérieur.

Guillaume, G. (1975 [1919]). Le problème de l'article et sa solution dans la langue française, Paris \& Québec : Librairie A.-G. Nizet \& Les Presses de l'Université Laval.

Halliday, M. A. K. et Hasan, R. (1976). Cohesion in English. Londres et New York : Longman.

Kleiber, G. (2001). L'anaphore associative. Paris : Presses Universitaires de France.

Kupferman, L. (1991). L'aspect du groupe nominal et l'extraction de en. Le français moderne, 59(2), 113-147.

Leeman, D. (2004). Les déterminants du nom en français : syntaxe et sémantique. Paris : Presses Universitaires de France.

Lefeuvre, F. (1999a). La phrase averbale en français. Paris : L'Harmattan.

Lefeuvre, F. (1999b). Les "marqueurs de prédication" dans la phrase averbale en français. Verbum, XXI, 4, 429-438.

Lefeuvre, F. (2005). Le prédicat nominal dans des articles de presse. in Syntaxe et sémantique 2005/1 ( $\left.{ }^{\circ} 6\right), 181-198<$ https://www.cairn.info/revue-syntaxe-et-semantique-2005-1-page-181.htm>.

Moignet, G. (1981). Systématique de la langue française. Paris : Klincksieck.

Naganuma, K. (2004). Furansugo ni okeru yuuhyoo no meishi gentei no bunpoo. Tokyo: Sobishuppansha.

Noailly, M. (1990). Le substantif épithète. Paris, Presses Universitaires de France.

Ohga, M. (1978). Comment on écrit en français (Gendai hutsusakubun no tekunikku). Tokyo: Taishukan.

Ohga, M. (1989). Ecrire pour mieux lire (Hôhô to shiteno Futsusakubun.). Tokyo, Hakusuisha.

Picabia L. (1983). Remarques sur le déterminant zéro dans les séquences en il y a. Le français moderne, 51(2), 157-171.

Picabia, L. (1986). Il y a démonstration et démonstration: réflexion sur l'article zéro. Langue française, 72, 80-101.

Picabia, L. (1991). Article zéro et structures apposées. Langage, 102, 88-102. 
Pottier, B. (1962). L'absence d'article en français et sa motivation. Revue de linguistique romane, 26, 158-162.

Rabatel, A. (2000). Valeurs représentative et énonciative du «présentatif » c'est et marquage du point de vue. Langue française, 128, 52-73.

Riegel, M., Pellat, J.-C. et Rioul, R. (1994). Grammaire méthodique du français. Paris : Presses Universitaires de France. 